\section{Percutaneous Endoscopic Lumbar Discectomy Under Local Anesthesia in Elderly Patients with Combined Spinal Canal Stenosis and Herniated Nucleus Pulposus}

\section{Abstract}

Percutaneous endoscopic discectomy (PED) for the lumbar spine was established in the early 2000s. This surgery can be performed under local anesthesia, and results in minimum damage to the back muscles. Thus, it is considered the least invasive disc surgery. Theoretically, this minimally invasive technique should be suitable for elderly patients who have comorbidities. However, such elderly patients usually also have foraminal stenosis due to facet hypertrophy, which makes the procedure technically demanding. Here, we have reported three cases of patients aged over 65 years who underwent PED. We first performed foraminoplasty using a highspeed drill and inserted an $8 \mathrm{~mm}$ cannula safely through the enlarged foramen. In all cases, the herniated fragments of the nucleus pulposus were successfully removed endoscopically under local anesthesia without any surgery-related complications. In all patients, low back and leg pain improved immediately postoperatively. In conclusion, even in elderly patients with foraminal stenosis, PED can be safely performed by utilizing the foraminoplasty technique. For elderly populations with severe comorbidities, this technique is expected to be especially useful.

Keywords: Percutaneous endoscopic discectomy; Elderly patients; Herniated nucleus pulposus; Foraminoplasty; Lumbar spine; Disc surgery; Computed tomography; Muscle; Hypertrophy; Anesthesia

Received: July 11, 2016; Accepted: April 12, 2017; Published: April 16, 2017

\author{
Tatsuhiko Henmi", \\ Tomoya Terai ${ }^{1}$, \\ Mitsunobu Abe², \\ Kazuta Yamashita², \\ Kosaku Higashino², \\ Toshinori Sakai \\ Yoichiro Takata ${ }^{2}$, \\ Fumitake Tezuka², \\ Akihiro Nagamachi² and
Koichi Sairyo $^{2}$
}

1 Department of Orthopaedic Surgery, Tokushima Prefecture Naruto Hospital, Japan

2 Department of Orthopaedics, Tokushima University Graduate School of Medicine, Japan

Corresponding author: Koichi Sairyo

” sairyokun@hotmail.com

Professor and Chairman, Tokushima University Graduate School of Medicine, Japan.

Tel: 81886337240

Fax: 81886330178

Citation: Henmi T, Terai T, Abe M, et al. Perc utaneous Endoscopic Lumbar Discectomy Under Local Anesthesia in Elderly Patients with Combined Spinal Canal Stenosis and Herniated Nucleus Pulposus. Spine Res. 2017, 3:1.

During the insertion of the cannula fitted with an endoscope through the intervertebral foramen, the most typical surgeryrelated complication is injury to the exiting nerve $[9,10]$. To avoid such complications in patients with foraminal stenosis, the foraminoplasty technique was developed [11-13].

Degenerative lumbar spinal canal stenosis (LSS) is a common spinal disorder affecting the elderly population. At times, HNP may occur in combination with LSS, which is known as combined 
spinal canal stenosis [14]. When the patient's symptoms are mainly caused by HNP rather that LSS, PED is a possible option for surgical intervention. In patients with LSS, the facet joints are usually hypertrophic, which can induce foraminal stenosis. In such cases, the interlaminar(IL) approach is recommended. To remove the HNP fragment using IL approach, general or epidural anesthesia is essential since the nerve root [5] needs to retract during surgery. Following foraminoplasty, the foramen is enlarged, allowing the endoscope to be passed through the foramen without damage of the exiting nerve root. Thus, it is not difficult to assume that performing PED in patients with combined LSS may require concurrent foraminoplasty to safely access the HNP and the epidural space.

In this paper, we describe three cases of patients aged over 65 years with combined LSS, who were successfully treated by PED performed along with foraminoplasty under local anesthesia.

\section{Case Reports}

\section{Case 1: An 86-year-old male patient}

The patient reported a history of sudden low back pain and left leg pain without a specific trigger. The VAS score was 8.6 for the low back pain and 10 for the leg pain (out of 10). The pain was so severe that the patient was unable to walk. After undergoing 1 month of conservative treatment, he was referred to us for surgical treatment because of no improvement in the pain. Neurological examination revealed a positive straight leg raising test at $70^{\circ}$ on the left side. Muscle weakness as evaluated by muscle motor testing was found in the tibialis anterior $(2 / 5)$, extensor hallucis longus (3/5), and flexor hallucis longus (3/5) muscles. Abnormal tendon reflexes were not noted. Reduced sensation was obvious over the dermatome area for the $L 5$ nerve root on the left leg.

MRI obtained at the first visit indicated the presence of HNP and moderate LSS at the L4-L5 level (Figure 1). The HNP was large, nearly reaching the central portion of the canal. Similar to case \#1, foraminal stenosis due to hypertrophy of the superior articular process of L5 was noted (Figure 1, right panel). Under local anesthesia using $1 \%$ xylocaine, PED was initiated in the prone position. The surgical procedure has been described in detail previously [9]. An $8 \mathrm{~mm}$ skin incision was made. Following the foraminotomy, the intervertebral foramen was enlarged, so that the cannula was safely and appropriately placed just beneath the HNP fragment. Following foraminoplasty, PED was carried out, and the HNP fragment was successfully removed. Figure 2 shows the MRI before and after PED surgery. Just after the surgery, the pain disappeared and the VAS scores decreased to 0.5 and 0 for low back and leg pain, respectively. By 1 month postoperatively, the muscle weakness had also improved. Foraminoplasty was evaluated using CT scans obtained before and after the surgery (Figure 3). Seventeen months after surgery, visual analog scale scores for low back pain and leg pain were $0 / 10$ and $0 / 10$, respectively.

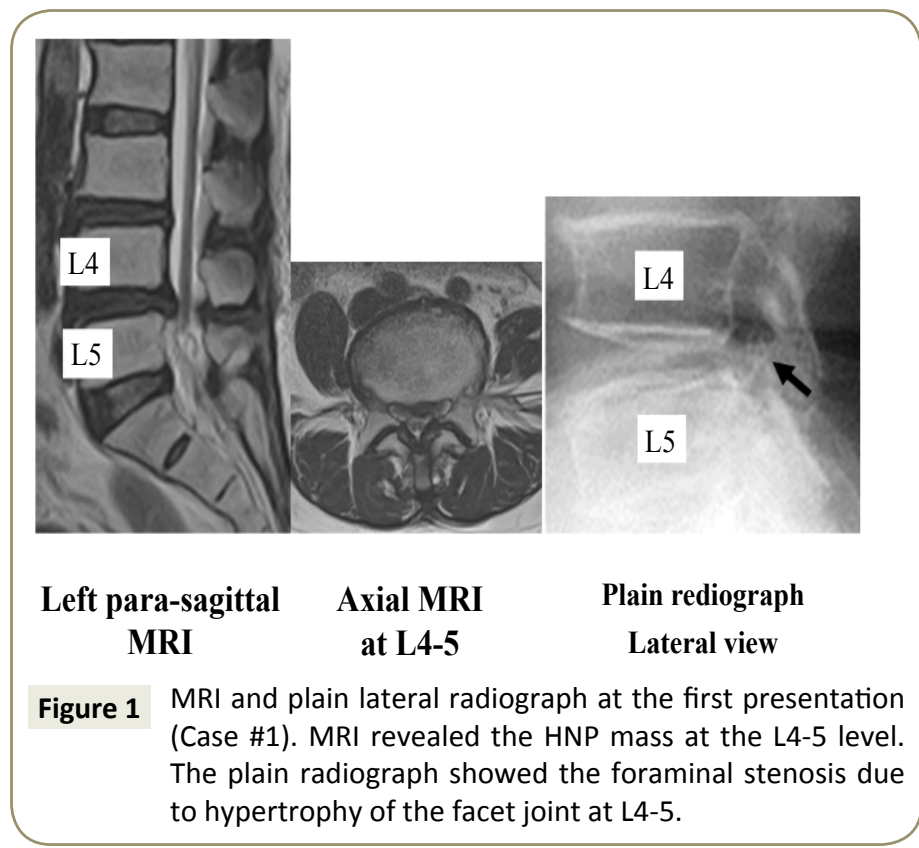

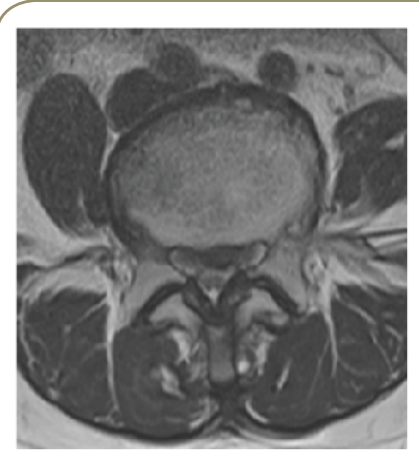

Prcopcrative

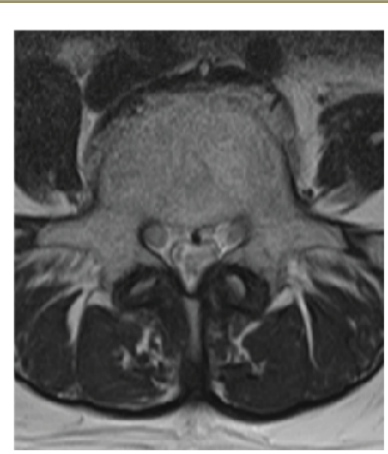

Postopcrative
Figure 2 MRI before and after the PED surgery (Case \#1). The HNP mass was successfully removed endoscopically.

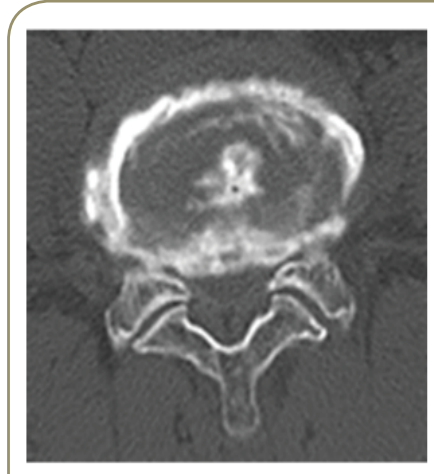

Prcopcrative

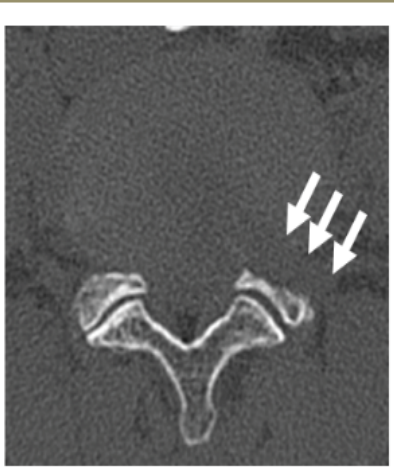

Postopcrative
Figure 3 CT discography before surgery and plain CT after the PED surgery (Case \#1). The arrows indicate the location of the foraminoplasty. 


\section{Case 2: A 77-year-old female patient}

This patient presented with pain in the left buttock and leg. One month before presentation, she had carried a heavy item, following which the symptoms appeared. First, she was treated by a primary care physician with NSAIDs. Neurological examination revealed a positive straight leg raising test at $70^{\circ}$ on the left side. No muscle weakness or abnormal tendon reflexes was noted. However, reduced sensation was obvious over the dermatome area for the L5 nerve root on the left leg. Magnetic resonance imaging (MRI) at the first visit indicated HNP and moderate LSS at the level of L4-L5 (Supplementary Figure 1). For one month, we treated the patient conservatively by changing the medication to gabapentin and performing a selective nerve root block, but the treatment was not effective in reducing her pain. The visual analog scale (VAS) score for leg pain was 7.2 out of 10 , and the pain disturbed her activities of daily living. Therefore, surgical treatment was planned, and a transforaminal approach for PED was selected.

A lateral plain radiograph (Supplementary Figure 1, right panel) revealed foraminal stenosis caused by hypertrophy of the superior articular process of L5. The HNP fragment was successfully removed using the full endoscopic technique. Supplementary Figure $\mathbf{2}$ shows the MRI before and after PED surgery. Although the LSS persisted, the HNP was completely removed. Immediately postoperatively, the pain disappeared and the VAS score decreased to 0 out of 10 . Foraminoplasty was evaluated using computed tomography (CT) images obtained before and after the surgery (Supplementary Figure 3).

Two years after surgery, visual analog scale scores for low back pain and leg pain were $0 / 10$ and $0 / 10$, respectively.

\section{Case 3: A 69-year-old male patient}

This patient had a history of chronic back pain for 10 years. Ten months before presentation, the patient experienced severe low back pain and left thigh pain during a golf swing. The VAS scores were 5 and 6 for low back and leg pain, respectively. The patient had a history of angina and was on anti-coagulation therapy. First, conservative treatment was administered; however, it was not effective. After 10 months, he was referred to us for minimally invasive surgical treatment. Neurological examination did not show any specific neurological abnormalities in motor function, sensory function, or deep tendon reflexes.

MRI at the first visit indicated a huge HNP and multilevel LSS at the L2-L3 level (Supplementary Figure 4). The HNP was large and nearly reached the central portion of the canal. Similar to cases \#1 and \#2, (Supplementary Figure 4, right panel), foraminal stenosis caused by hypertrophy of the superior articular process of L3 was noted. Discography was conducted for a better understanding of the pain source in this patient, and concordant low back and leg pain was confirmed. Following foraminotomy, PED was performed and the HNP fragment was successfully removed. Supplementary Figure $\mathbf{5}$ shows the MRI before and after the PED surgery. Although LSS persisted, the HNP was completely removed. Immediately postoperatively, the pain decreased; at
3 months postoperatively, the VAS score had reduced to 1 for both low back and leg pain. Foraminoplasty was evaluated using CT scans obtained before and after the surgery (Supplementary Figure 6).

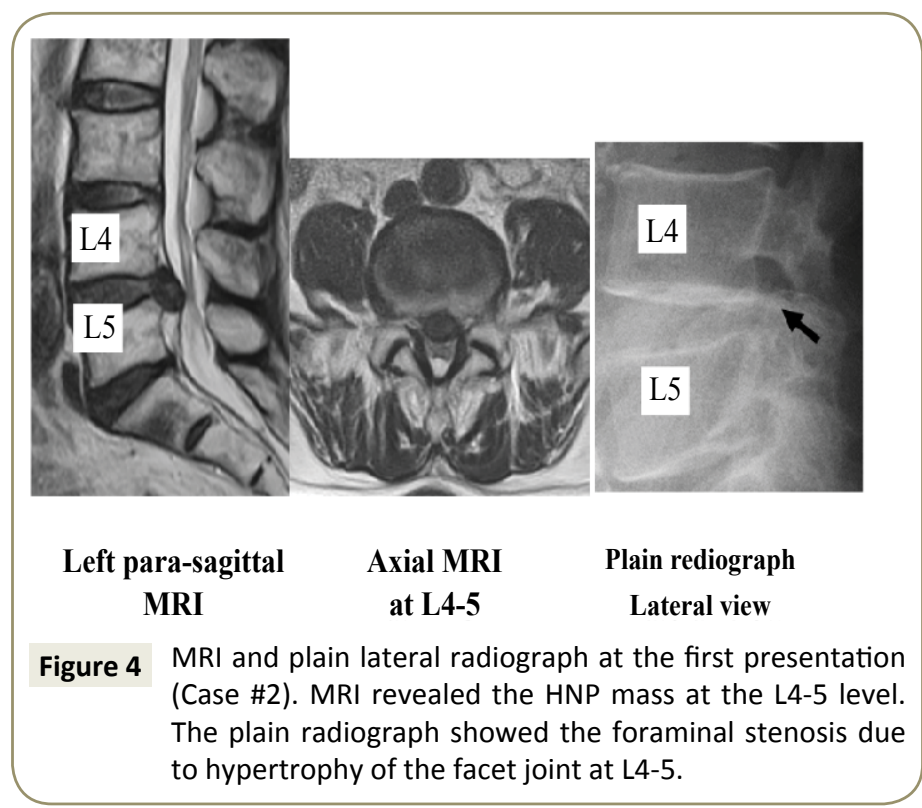

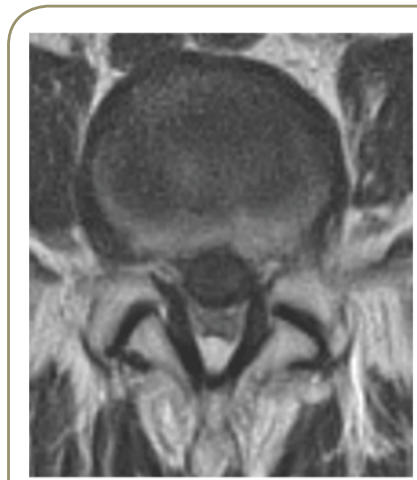

\section{Prcopcrative}

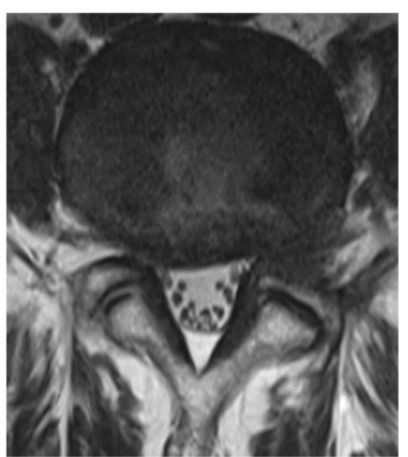

Postopcrative
Figure $5 \mathrm{MRI}$ before and after the PED surgery (Case \#2). The HNP mass was successfully removed endoscopically.

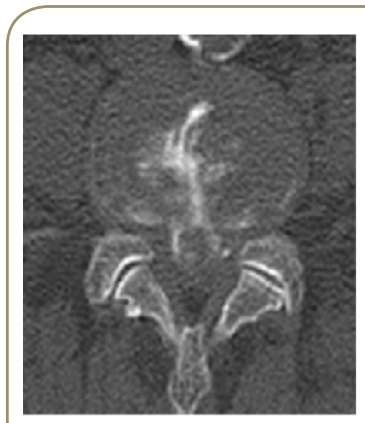

Prcopcrative

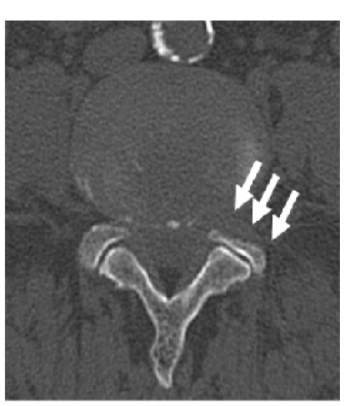

Postopcrative
Figure 6 CT discography before surgery and plain CT after the PED surgery (Case \#2). The arrows indicate the location of the foraminoplasty. 


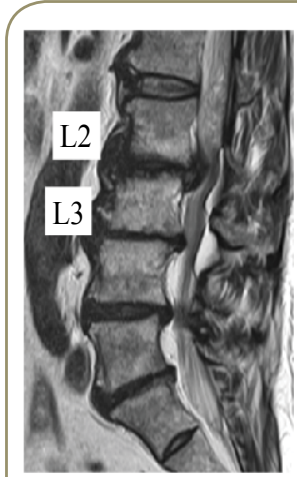

Left para-sagittal MRI
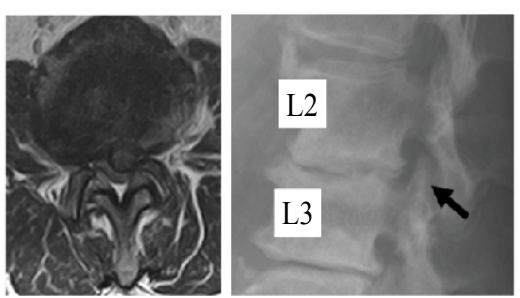

\section{Axial MRI at L2-3}

\section{Plain radiograph Lateral view}

Figure $7 \mathrm{MRI}$ and plain lateral radiograph at the first presentation (Case \#3). MRI revealed the HNP mass at the L2-3 level. The plain radiograph showed the foraminal stenosis due to hypertrophy of the facet joint at L2-3.

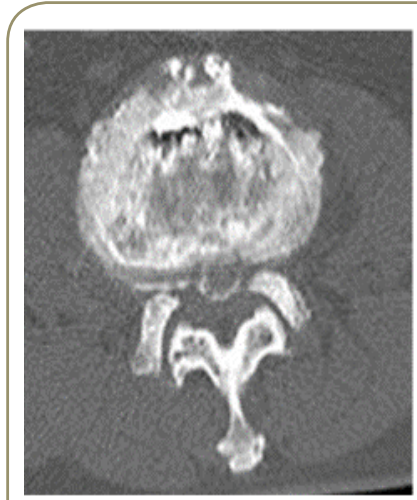

Prcopcrative

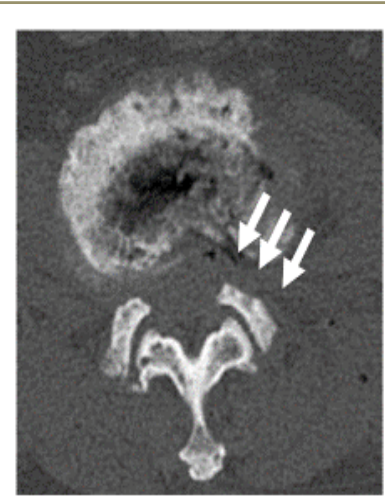

Postopcrative
Figure 8 MRIs before and after the PED surgery (Case \#3). The HNP mass was successfully removed endoscopically.
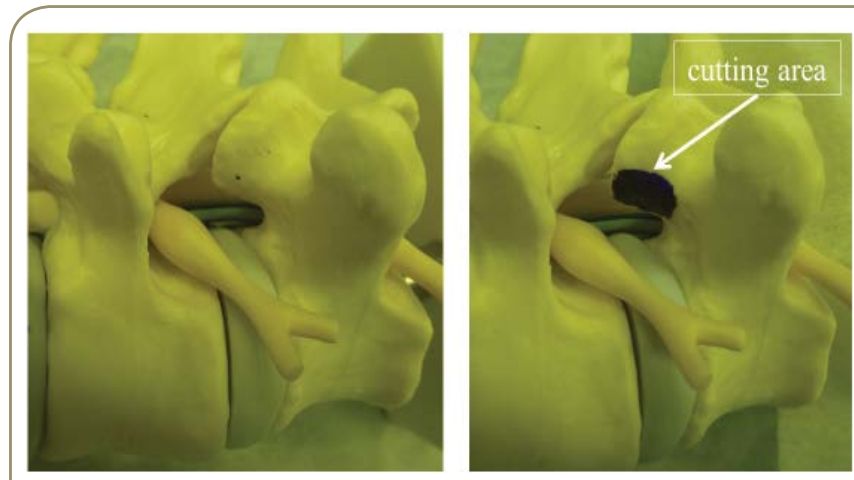

Figure 9 CT discography before surgery and plain CT after the PED surgery (Case \#3). The arrows indicate the location of the foraminoplasty.

\section{Discussion}

The procedure of PED for the lumbar spine was established in the year 2000, owing to the tremendous efforts of several research groups [2-4,15,16]. After appropriate surgical instruments for PED were developed, the indications for PED increased. In particular, the development of the high-speed drill, which can be used within the small cannula for PED, made bone decompression possible. Dezawa et al. reported a unique PED technique for removal of HNP in the hidden zone using the high-speed drill [6]. HNP that had migrated to the hidden zone can be removed through the bone hole of the lamina, and this technique is called the percutaneous endoscopic translaminar approach [6]. Abe et al. [12]. described the transforaminal PED technique at the L5-S level. They stated that the cannula can be passed through the foramen following enlargement by foraminoplasty using the high-speed drill. More recently, Henmi et al. [13] clearly described the ventral epiduroscopic observation technique following foraminotomy using a video presentation (Figures 4-10).

In this paper, we have presented three cases of lumbar HNP combined with LSS. This pathology is a good indication for decompression using surgical loupe or microscope [14]. As minimally invasive procedures, a tubular type retractor and spinal endoscope have also been used for decompression in this disorder $[17,18]$. For our three cases, the main symptom was severe leg pain occurring even at rest. Thus, discectomy rather than laminectomy was considered important for relieving this symptom. PED, which is also called full endoscopic discectomy, is a minimally invasive disc surgery, since it requires only an 8 $\mathrm{mm}$ skin incision and can be performed under local anesthesia. Furthermore, during the procedure, damage to the major back muscles is also minimal, and it is thus suitable for athletes $[18,12,19]$.

The interlaminar approach has been performed at L5-S1 with high iliac crest associated with wide inter laminar space and relatively small t [20] hecal sac. This interlaminar technique can use either the axilla approach or the shoulder approach. The axilla approach is used if there is no safe area in the axilla region. These two approaches are compatible. However, the interlaminar approach has several disadvantages. For instance, the narrow interlaminar space can recult in dysesthesia and pain, as well as requiring

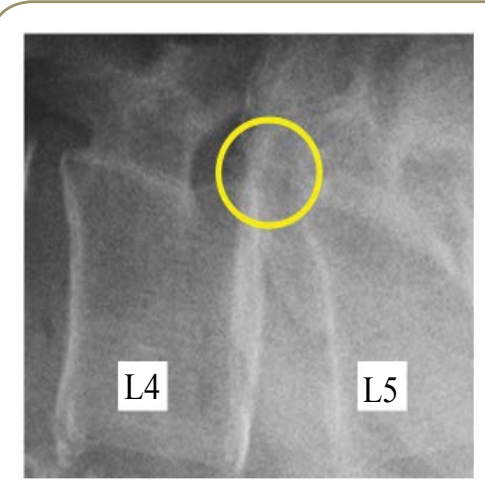

\section{Location of the endoscope}

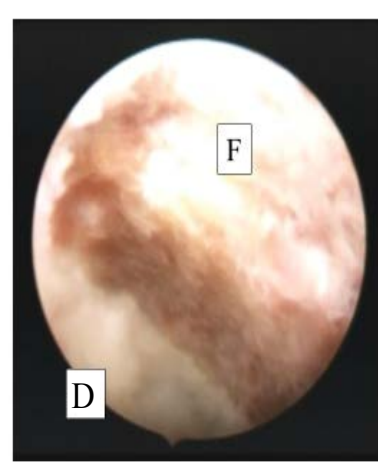

Endoscopic view
Figure 10 Plain radiograph and endoscopic view (Case \#2). In the right panel showing the endoscopic view, the superior articular process of L5 is clearly observed to be drilled and shaved into a round shape. F: facet, D: disc 
expensive surgical instruments. The elderly patients usually have lateral recess stenosis; it is difficult the axilla approach. Choi reported that open microdiscectomy should be considered if preoperative neuroimaging detects no safe spaces in either the shoulder or axilla regions [20].

The intervertebral foramen is usually narrow in elderly patients with LSS due to facet joint hypertrophy (Figure 1 and Supplementary Figures 1 and $\mathbf{4}$ ), unlike that in young patients. If the $8 \mathrm{~mm}$ cannula is inserted through such a narrowed foramen, impingement of the exiting nerve root would be unavoidable $[9,10]$. Actually, in all our cases, the transverse diameter of the foramen at the disc level was less than $8 \mathrm{~mm}$. In such cases, foraminoplasty with a high-speed drill should be performed with the standard PED procedure. Figure 4 illustrates a radiograph and real endoscopic view during the foraminoplasty in case 1 . In the right panel showing the endoscopic view, the superior articular process of $\mathrm{L} 5$ is clearly noted to be drilled and shaved into a round shape, so that the cannula can be inserted smoothly. Following the foraminoplasty, the narrowed foramen was enlarged (Figure 3 and Supplementary Figures 3 and 6), and the $8 \mathrm{~mm}$ cannula could be safely inserted into the spinal canal. The HNP was then successfully removed without any complications.

LSS is one of the common spinal disorders in the elderly population. In this population, many patients have comorbidities, such as heart disease, pulmonary dysfunction, kidney disorders. In such cases, general anesthesia is often difficult. The PED procedure can be performed under local anesthesia, which is the biggest advantage of this technique. In 2013, Kitahama et al. [7] reported a case of LSS with severe lung comorbidities treated surgically under local anesthesia with the PED technique; consequently, the authors commented on the usefulness of the PED technique especially in elderly patients in a poor general condition. Similarly, as described in this case report, we could successfully treat three elderly patients aged over 65 years under local anesthesia. Considering Japan's aging population, the patient population with comorbidities is expected to increase. Therefore, this minimally invasive PED procedure performed under local anesthesia is expected to become increasingly important.

\section{Conclusion}

In conclusion, we presented three cases of HNP combined with LSS in elderly patients who were treated with foraminoplasty and PED for complete removal of the HNP. We consider that this minimally invasive procedure performed under local anesthesia is an important option for the surgical treatment of HNP in elderly patients, especially those with severe comorbidities. 


\section{References}

1 Hijikata S (1989) Percutaneous nucleotomy. A new concept technique and 12 years' experience. Clin Orthop Relat Res 238: 9-23.

2 Yeung A (2000) The evolution of percutaneous spinal endoscopy and discectomy: State of the art. Mt Sinai J Med 67: 327-332.

3 Yeung A, Tsou PM (2002) Posterolateral endoscopic excision for lumbar disc herniation: Surgical technique, outcome, and complications in 307 consecutive cases. Spine 27: 722-731.

4 Yeung A, Yeung CA (2007) Minimally invasive techniques for the management of lumbar disc herniation. Orthop Clin North Am 38: 363-372.

5 Dezawa A, Sairyo K (2011) New minimally invasive endoscopic discectomy technique through the interlaminar space using a percutaneous endoscope. Asian J Endosc Surgery 4: 94-98.

6 Dezawa A, Mikami H, Sairyo K (2011) Percutaneous endoscopic translaminar approach for herniated nucleus pulposus in the hidden zone of the lumbar spine. Asian J Endosc Surgery 5: 200-203.

7 Kitahama Y, Sairyo K, Dezawa A (2013) Percutaneous endoscopic transforaminal approach to decompress the lateral recess in an elderly patient with spinal canal stenosis, herniated nucleus pulposus and pulmonary comorbidities. Asian J Endosc Surg 6: 130-133.

8 Sairyo K, Kitagawa Y, Dezawa A (2013) Percutaneous endoscopic discectomy and thermal annuloplasty for professional athletes. Asian J Endosc Surg 6: 292-297.

9 Sairyo K, Egawa H, Matsuura T, Takahashi M, Higashino K, et al. (2014) State of the art: Transforaminal approach for percutaneous endoscopic lumbar discectomy under local anesthesia. J Med Invest 61: 217-225.

10 Sairyo K, Matsuura T, Higashino K, Sakai T, Takata Y, et al. (2014) Surgery related complications in percutaneous endoscopic lumbar discectomy under local anesthesia. J Med Invest 61: 264-269.
11 Lee SH, Kang HS, Choi G, Kong BJ, Ahn Y, et al. (2010) Foraminoplastic ventral epidural approach for removal of extruded herniated fragment at the L5-S1 level. Neurol Med Chir 50: 1074-1078.

12 Abe M, Takata Y, Higashino K (2015) Foraminoplastic transforaminal percutaneous endoscopic discectomy at the lumbosacral junction under local anesthesia in an elite rugby player. J Med Invest 62: 238-241

13 Henmi T, Terai T, Hibino N (2015) Percutaneous endoscopic lumbar discectomy utilizing ventral epiduroscopic observation technique and foraminoplasty for transligamentous extruded nucleus pulposus: technical note. J Neurosurg Spine 13: 1-6

14 Mayer HM, List J, Korge A (2003) Microsurgery of acquired degenerative lumbar spinal stenosis. Bilateral over-the-top decompression through unilateral approach. Orthopade 32: 889-895

15 Kambin P, Schaffer JL (1989) Percutaneous lumbar discectomy. Review of 100 patients and current practice. Clin Orthop Relat Res 24-34.

16 Schreiber A, Leu H (1991) Percutaneous nucleotomy: technique with discoscopy. Orthopedics 14: 439-444.

17 Sairyo K, Sakai T, Higashino K, Inoue M, Yasui N, et al. (2010) Complications of endoscopic lumbar decompression surgery. Minim Invasive Neurosurg 53: 175-178.

18 Wada K, Sairyo K, Sakai T, Yasui N (2010) Minimally invasive endoscopic bilateral decompression with a unilateral approach (endo-BiDUA) for elderly patients with lumbar spinal canal stenosis. Minim Invasive Neurosurg 53: 65-68.

19 Yamashita T, Higashino K, Sakai T (2016) Revision percutaneous lumbar endoscopic discectomy under the local anesthesia for the recurrent lumbar herniated nucleus pulposus in a high-class athlete: a case report. J Med Invest 63: 135-139.

20 Choi G, Lee SH, Raiturker PP, Lee S, Chae YS (2006) Percutaneous endoscopic interlaminar discectomy for intracanalicular disc herniations at L5-S1 using a rigid working channel endoscope. Neurosurgery 58: 59-68. 